\title{
Antagonistic Effects of Enrofloxacin on Carbendazim-Induced Developmental Toxicity in Zebrafish Embryos
}

\author{
Ruiqi Fan ${ }^{1,2}$, Wanjun Zhang ${ }^{1,2}$, Li Jia ${ }^{2}$, Sunlin Luo ${ }^{1}$, Ying Liu ${ }^{1}$, Yongpeng Jin ${ }^{1}$, Yongchen Li ${ }^{1}$, Xiaoyan Yuan ${ }^{2,3}$ \\ and Yiqiang Chen ${ }^{1, *}$ \\ 1 State Key Laboratory of Animal Nutrition, College of Animal Science and Technology, China Agricultural \\ University, Beijing 100193, China; fanruiqi94110@cau.edu.cn (R.F.); wanjunzhang@cau.edu.cn (W.Z.); \\ 1s118810791522@163.com (S.L.); liuyingcau2021@163.com (Y.L.); jinyp@cau.edu.cn (Y.J.); \\ liyongc2021@163.com (Y.L.) \\ 2 Center of Disease Control and Prevention, PLA, Beijing 100073, China; jiali1230@aliyun.com (L.J.); \\ yanziyuan2007@126.com (X.Y.) \\ 3 School of Nursing and Health, Henan University, Kaifeng 475000, China \\ * Correspondence: yqchen@cau.edu.cn
}

Citation: Fan, R.; Zhang, W.; Jia, L.; Luo, S.; Liu, Y.; Jin, Y.; Li, Y.; Yuan, X.; Chen, Y. Antagonistic Effects of Enrofloxacin on CarbendazimInduced Developmental Toxicity in Zebrafish Embryos. Toxics 2021, 9, 349. https://doi.org/10.3390/toxics 9120349

Academic Editor: Robyn L. Tanguay

Received: 22 October 2021

Accepted: 29 November 2021

Published: 10 December 2021

Publisher's Note: MDPI stays neutral with regard to jurisdictional claims in published maps and institutional affiliations.

Copyright: (c) 2021 by the authors. Licensee MDPI, Basel, Switzerland. This article is an open access article distributed under the terms and conditions of the Creative Commons Attribution (CC BY) license (https:// creativecommons.org/licenses/by/ $4.0 /)$.

\begin{abstract}
Carbendazim (CAR) and enrofloxacin (ENF) are frequently detected in fruits and meat products, respectively. Since most people consume fruits, vegetables, and meat products, combined exposure is possible, necessitating further evaluation of toxic interactions. In this study, the developmental toxicity of separate and combined exposure was examined in zebrafish embryos. Carbendazim exposure at $0.79 \mathrm{mg} / \mathrm{L}$ and above significantly affected developmental parameters, while enrofloxacin alone had no substantial effects on these developmental parameters within the selected concentration range (0.10-0.40 mg/L). Surprisingly, ENF antagonized the CAR-evoked reduction in the $48 \mathrm{hpf}$ (hours post-fertilization) hatching rate and the increases in the $96 \mathrm{hpf}$ malformation and lethality rates. The results revealed that the antagonism might be associated with reciprocal effects of these compounds on metabolism-related genes, such as cyp $7 a 1$ and apoa1a. These results reveal a complex interaction between ENF and CAR on metabolic regulation during development and highlight the importance of combined assessment for agents with the potential for simultaneous exposure.
\end{abstract}

Keywords: carbendazim; enrofloxacin; zebrafish; embryonic developmental toxicity; antagonism

\section{Introduction}

Enrofloxacin (ENF) is a fluoroquinolone antibiotic widely used in animal production and generally considered safe for livestock and of low exposure risk to agricultural workers. However, recent reports indicate that long-term use may result in relatively high residual levels in the eggs of treated hens [1,2]. Further, ENF treatment of mares was reported to damage foetal cartilage [3]. Additionally, low doses of ENF to mice can cause changes in the intestinal flora and induce bacterial resistance [4]. Collectively, these studies indicate that the risks of ENF to human health warrant further evaluation.

Carbendazim (CAR) is a broad-spectrum benzimidazole fungicide prohibited in the United States due to substantial toxicity. However, it is still used on a large scale in several other countries, and there are reports of residual levels in excess of allowable limits on various fruits and vegetables grown in China [5]. Studies have confirmed that CAR can interfere with endocrine function [6] and reproductive function [7] in rats.

In modern agricultural practice, multiple pesticides, fungicides, and antibiotics may be used to protect crops and livestock, increasing the risk of simultaneous exposure and unexpected toxic interactions. Most reports on mixed toxicity concern multiple pesticides [8,9] or antibiotics $[10,11]$, while there has been relatively little study on the interactions between crop pesticides and veterinary antibiotics. CAR is one of the most frequently detected 
pesticides in China [12], and ENF is on the list of the most frequently detected veterinary antibiotics in China [13]. Given that most people consume animal products, fruits, and vegetables daily, a comprehensive human health risk assessment should include cross-category toxicity. Moreover, the cross-category toxicity of CAR and ENF still lacks investigation, and it is necessary to explore the combined toxicity of the two chemicals.

Zebrafish share a high degree of genetic homology with humans and have the advantage of integrating in vitro cells and in vivo animal models [14]. The zebrafish model is of high efficiency and low cost. In recent years, it has been widely used in the field of drug research and development for the toxicity evaluation of compounds and the highthroughput screening of active compounds. Thus, zebrafish embryos were selected as the model of our research [15].

Large-scale gene expression profiling by transcriptomics can help reveal the molecular mechanisms of joint toxicity $[16,17]$. In this study, the individual and combined effects of CAR and ENF were examined on embryonic zebrafish development, followed by transcriptomics with qPCR verification of gene expression changes for preliminary examination of the underlying molecular mechanisms. We intended to explore the complex interaction between ENF and CAR on metabolic regulation during development, and highlight the importance of combined assessment for agents with the potential for simultaneous exposure.

\section{Materials and Methods}

\subsection{Reagents}

Carbendazim (99\%) was purchased from MedChemExpress (Monmouth Junction, NJ, USA), enrofloxacin ( $>98 \%$ ) from Sigma-Aldrich (Saint Louis, MO, USA), and DMSO $(99.7 \%)$ from Thermo Fisher (Waltham, MA, USA). All other reagents were of analytical grade and purchased from Shanghai Sinopharm Group Chemical Reagents.

\subsection{Zebrafish Breeding and Egg Collection}

Wild-type Tu zebrafish were purchased from Wuhan Zebrafish Resource Center (Wuhan, China) and maintained in a dedicated breeding system from ESEN Technology (Beijing, China) under a $14 \mathrm{~h} / 10 \mathrm{~h}$ light cycle and ambient room temperature of $28 \pm 2{ }^{\circ} \mathrm{C}$. Animals were fed freshly hatched brine shrimp larvae twice daily. In the afternoon before experiments, two male and two female fish were placed in separate compartments of a breeding box. At the beginning of the photoperiod the following day, the partition separating compartments was removed to allow the males and females to chase freely. After mating, the fertilized eggs were collected within $30 \mathrm{~min}$.

\subsection{Zebrafish Embryo Exposure and Developmental Index Detection}

Pesticides were weighed, dissolved in DMSO as stock solutions, and diluted in Holt buffer (containing $\mathrm{NaCl} 3.5 \mathrm{~g} / \mathrm{L}, \mathrm{KCl} 0.05 \mathrm{~g} / \mathrm{L}, \mathrm{CaCl}_{2} 0.1 \mathrm{~g} / \mathrm{L}, \mathrm{NaHCO}_{3} 0.05 \mathrm{~g} / \mathrm{L}$ ) to the indicated treatment concentrations. The final DMSO concentration $(0.1 \%)$ had no detectable effects on zebrafish development in published papers [18,19]. The final ENF concentrations were $0.10,0.13,0.16,0.20,0.26,0.32$, and $0.40 \mathrm{mg} / \mathrm{L}$, and the final CAR concentration were $0.50,0.63,0.79,1.00,1.26,1.59$, and $2.00 \mathrm{mg} / \mathrm{L}$. The concentrations selected were based on the maximum residual levels of CAR (0.05-3 mg $/ \mathrm{kg}$ [20]) and ENF (0.1-0.3 mg/kg [21]) in food in China. The mixed exposure liquid (MIX) (ENF: CAR $=1: 4.84, v / v$ ) was prepared according to the acceptable daily intake (ADI) from the Ministry of Agriculture and Rural Affairs of the People's Republic of China [20,21]. Total MIX concentrations were $0.54,0.68$, $0.86,1.09,1.37,1.72$, and $2.17 \mathrm{mg} / \mathrm{L}$. The CAR concentrations in MIX were 0.09, 0.12, 0.15, $0.19,0.23,0.29$, and $0.37 \mathrm{mg} / \mathrm{L}$, and the ENF concentrations in MIX were 0.45, 0.56, 0.71, $0.90,1.14,1.43$, and $1.80 \mathrm{mg} / \mathrm{L}$.

Eggs were collected at $4 \mathrm{hpf}$ (hours post-fertilization) and examined under an SZ-10 stereomicroscope (Olympus, Japan) to remove unfertilized, coagulated, and damaged samples. Twenty healthy eggs were placed in each well of a 6-well plate and treated 
with $10 \mathrm{~mL}$ of the indicated solution, with three replicates at each concentration. The solution was exchanged every $24 \mathrm{~h}$ for $96 \mathrm{~h}$ and dead eggs were removed at each exchange. Embryonic hatching, mortality, and malformation rates of each treatment group were recorded at $24,48,72$, and $96 \mathrm{hpf}$.

\subsection{Exposure for Transcriptomics}

Exposure concentrations for transcriptomics were set according to the benchmark doses (BMDL 10 values) of ENF alone, CAR alone, and the mixture. Fertilized eggs were treated from $4 \mathrm{hpf}$ to $96 \mathrm{hpf}$, with three biological replicates per treatment group and 80 eggs per replicate. After $96 \mathrm{hpf}$, treated eggs in each group were transferred to a $2 \mathrm{~mL}$ centrifuge tube, and the supernatant was discarded by centrifugation at 12,000 $\mathrm{rcf}$ and $4{ }^{\circ} \mathrm{C}$ for $10 \mathrm{~min}$. Larvae were then washed twice with $1 \mathrm{~mL}$ PBS, with centrifugation and supernatant removal between washes. RNA was extracted using Trizol reagent according to the manufacturer's instructions (Ambion, Austin, TX, USA) and stored at $-80{ }^{\circ} \mathrm{C}$ for further testing.

\section{5. mRNA Library Construction}

RIN (RNA integrity number) and concentration were assessed using Fragment Analyzer 5400 (Agilent, Santa Clara, CA, USA), and the RINs were all above 9. Oligo(dT)attached magnetic beads were used to purify mRNA. The First-strand cDNA was then generated through a random hexamer-primed reverse transcription, followed by secondstrand cDNA synthesis. The single-strand circle DNA (ssCir DNA) was formatted as the final library. The final library was amplified with phi29 to make DNA nanoballs (DNB), with more than 300 copies of one molecular. DNBs were loaded into the patterned nanoarray, and single-end 50 bases reads were generated on the BGIseq500 platform (BGIShenzhen, China). The transcriptome raw data were submitted to the NCBI database with the BioProject number PRJNA780808.

\subsection{RNA-Seq Data Analyze}

The filter of the sequencing data was with SOAPnuke [22]. Low-quality and unknown reads were removed. Then, clean reads were obtained and stored in FASTQ format. HISAT2 (v2.0.4) was used to map the clean reads to the reference genome [23]. Bowtie2 (v2.2.5) was applied to align the clean reads to the reference coding gene set [24]. The expression level of genes was calculated by RSEM (v1.2.12) [25]. The reference database was from NCBI (GRCz11). Essentially, differential expression analysis was performed using the DESeq2(v1.4.5) [26]. GO (http:/ / www.geneontology.org/, accessed on 6 July 2021) and KEGG (https:/ /www.kegg.jp/, accessed on 7 July 2021) enrichment analysis of annotated different expressed genes was then performed to explore the biological function further. The significant terms and pathways were corrected by a $p$-value with a rigorous threshold ( $p$-value $\leq 0.05$ ). The heatmap and GO network were completed through Hiplot (https:/ /hiplot.com.cn/, accessed on 5 October 2021).

\subsection{Real-Time Quantitative Reverse Transcription PCR}

To verify BGI-Seq results, RNA extracted as described was reverse transcribed into cDNA using a reverse transcription kit (Thermo Scientific, Waltham, MA, USA) with SYBR and the primer sequences listed in Supplementary Materials on an LightCycler480 II thermocycler (an LightCycler480 II thermocycler) instrument. The reaction volume was $25 \mu \mathrm{L}$ and the thermocycle program was $95^{\circ} \mathrm{C}$ for $3 \mathrm{~min}, 45$ cycles of $95^{\circ} \mathrm{C}$ for $5 \mathrm{~s}, 60^{\circ} \mathrm{C}$ for $30 \mathrm{~s}$, and dissociated according to instrument guidelines. Expression levels relative to $\beta$-actin as the reference gene were calculated using the $2^{-\Delta \Delta C T}$ method (primer pairs of selected genes can be found in Table S1). 


\subsection{Data Analysis and Statistics}

SPSS 22.0 was used for statistical analysis of all developmental parameters and to calculate the $\mathrm{LC}_{50}$ values. Developmental parameters and qPCR analysis results were expressed as mean \pm standard deviation. One-way analysis of variance (ANOVA) and Tukey's test were used to compare the means of multiple groups if they passed Levene's Test. $p$-values $<0.05$ were considered to be statistically significant. Graphpad 8.0 was used to draw $96 \mathrm{hpf}$ dose-response curves and BMDS 3.2 software (EPA, Washington, DC, USA) to calculate BMDL 10 values [27]. CompuSyn 1.0.4 software [28] was used to construct $96 \mathrm{hpf}$ concentration-lethality curves and concentration-deformation rate curves, and to calculate joint toxicity effects by combination index (CI) analysis. Transcriptomic results were analyzed using the BGI Dr.Tom online work platform (https: / biosys.bgi.com, accessed on 1 October 2021).

\section{Results}

\subsection{Toxicity of CAR Alone on Zebrafish Embryo Development}

Carbendazim significantly altered the hatching, deformity, and lethality rates of zebrafish embryos (Figure 1). At 48 and $72 \mathrm{hpf}$, low-dose CAR (0.50-0.63 mg/L) increased the hatching rate of zebrafish embryos, while high-dose CAR $(0.79-1.26 \mathrm{mg} / \mathrm{L})$ decreased the hatching rate at 48 and $72 \mathrm{hpf}$ (Figure 1A). Carbendazim also significantly and dosedependently enhanced deformation and lethality rates (Figure 1B,C). High-dose CAR $(1.00-2.00 \mathrm{mg} / \mathrm{L})$ significantly increased the deformity rate from 0 to $48 \mathrm{hpf}$, reaching $90 \%$ by 48 hpf. The primary malformations caused by high-dose CAR included pericardial oedema, yolk sac oedema, and spinal curvature (Figure 1D1). High-dose CAR (1.59-2.00 mg/L) also dramatically enhanced embryonic lethality between 0 and $48 \mathrm{hpf}$, reaching nearly $100 \%$ at $48 \mathrm{hpf}$, while medium-dose CAR $(0.79-1.26 \mathrm{mg} / \mathrm{L})$ eliminated more than $30 \%$ of embryos within this same period. Lethality and teratogenicity were not detected at concentrations below $0.63 \mathrm{mg} / \mathrm{L}$.

A

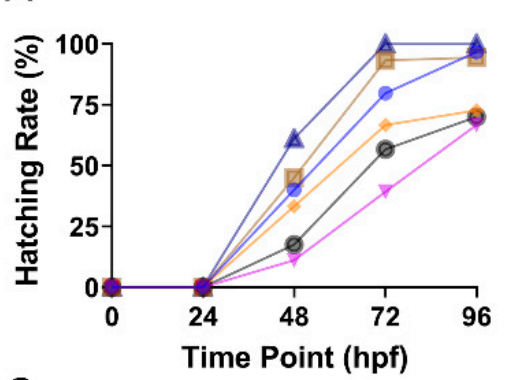

C

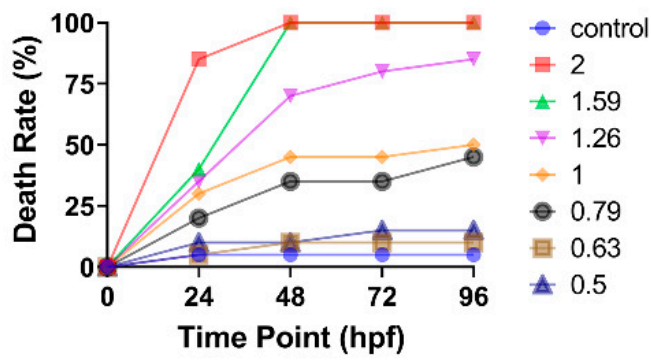

- control
$=2$
$=1.59$
-1.26
-1
-0.79
$\neq 0.63$
$\star 0.5$

B
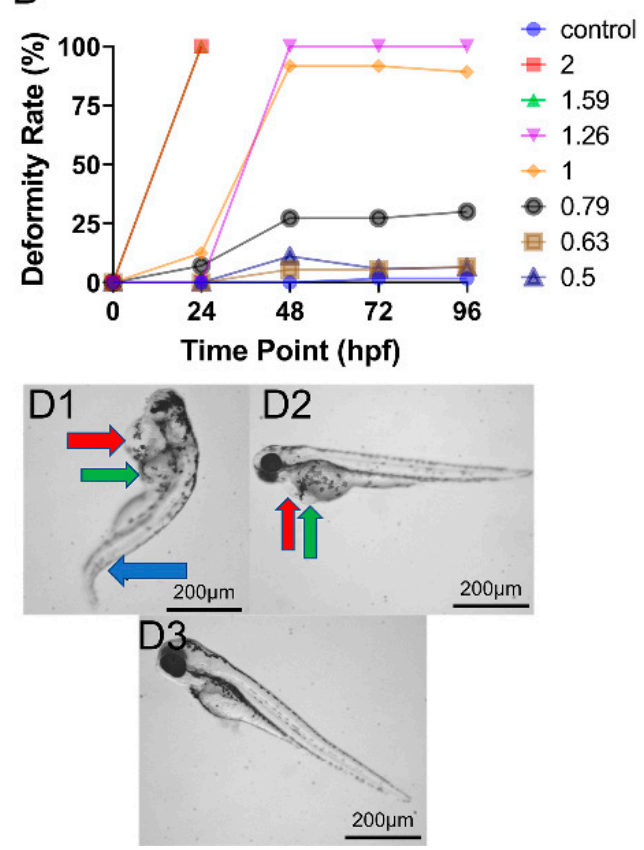

Figure 1. Concentration- and time-dependent effects of CAR on zebrafish embryo development $(n=60)$. (A) Time-response curves for hatching rate at all tested doses. (B) Time-response curves for deformity rate at all tested doses. (C) Timeresponse curves for death rate at all tested doses. (D) Typical deformities induced by (D1) $1.26 \mathrm{mg} / \mathrm{L}$, (D2) $1.00 \mathrm{mg} / \mathrm{L}$, and (D3) $0.50 \mathrm{mg} / \mathrm{L} \mathrm{CAR}$ at $96 \mathrm{hpf}$. Red arrows demarcate areas of pericardial oedema, green arrows demarcate regions of yolk sac oedema, and blue arrows show regions of spinal curvature. 


\subsection{Toxicity of ENF Alone on Zebrafish Embryo Development}

In general, ENF exhibited much lower developmental toxicity than CAR within the selected dose range (Figure 2). Enrofloxacin doses from 0.10 to $0.40 \mathrm{mg} / \mathrm{L}$ increased the hatching rate, especially from 24 to $72 \mathrm{hpf}$ (Figure 2A), but with relatively modest dose dependence. Further, teratogenicity and lethality were also modest within the selected dose range, even after 72-96 hpf (Figure 2B,C), and again were not strongly dose-dependent. Indeed, at $96 \mathrm{hpf}$, there were no significant differences in deformity and death rate among concentration groups. An increase in deformity rate was not substantial until 48 to $72 \mathrm{hpf}$, while mortality increased sooner (0-24 hpf) but remained lower than CAR-induced mortality even at high doses. The deformity rate of 0.13 and $0.32 \mathrm{mg} / \mathrm{L}$ ENF at $96 \mathrm{hpf}$ was lower compared to $72 \mathrm{hpf}$, which resulted from the death of deformed larvae during this period. The most common deformity in all dose groups was slight pericardial oedema (Figure 2D1).

A

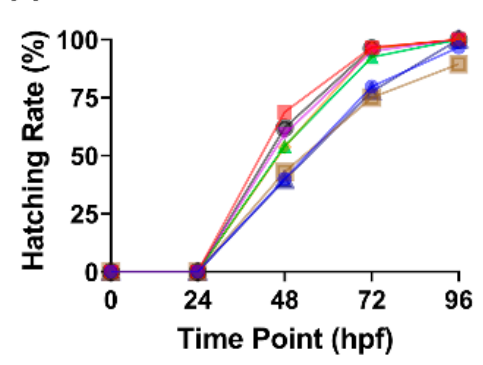

C

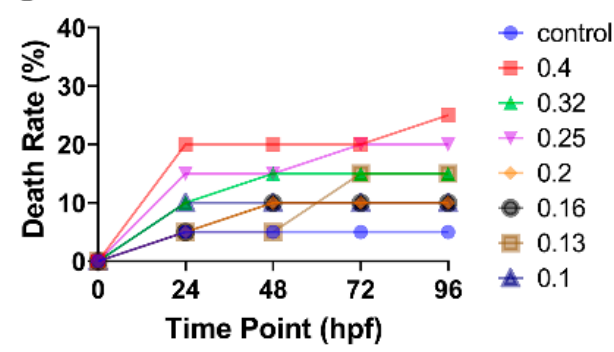

$\mathrm{B}$
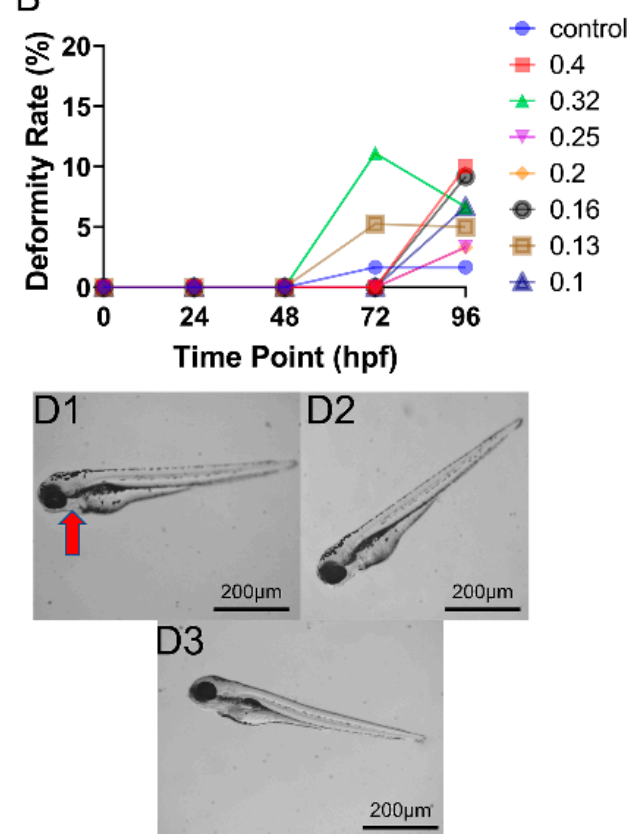

Figure 2. Concentration- and time-dependent effects of ENF on zebrafish embryo development $(n=60)$. (A) Time-response curves for hatching rate at all tested doses. (B) Time-response curves of deformity rate at all tested doses. (C) Timeresponse curves of death rate at all tested doses. (D) Typical deformities induced by (D1) $0.40 \mathrm{mg} / \mathrm{L}$, (D2) $0.20 \mathrm{mg} / \mathrm{L}$, and (D3) $0.10 \mathrm{mg} / \mathrm{L}$ ENF at $96 \mathrm{hpf}$. Red arrow indicates a region of pericardial oedema.

\subsection{Toxicity of Mixed CAR and ENF Exposure on Zebrafish Embryo Development}

The addition of ENF (MIX treatment) reduced the developmental toxicity of CAR (Figure 3). First, the hatching rate was higher under high-dose MIX than high-dose CAR at 48 to $72 \mathrm{hpf}$ (Figure $4 \mathrm{~A}$ ), while lower MIX doses had little influence on the hatching rate. Further, CAR concentrations that were teratogenic or lethal when applied alone were substantially less damaging when applied in MIX. For instance, MIX demonstrated substantial teratogenic and lethal effects only at $2.17 \mathrm{mg} / \mathrm{L}$ and $1.72 \mathrm{mg} / \mathrm{L}$, doses containing $1.81 \mathrm{mg} / \mathrm{L}$ and $1.43 \mathrm{mg} / \mathrm{L}$ CAR, but no substantial toxicity was observed at or below $1.37 \mathrm{mg} / \mathrm{L}$ MIX, which contained a dose of CAR $(1.14 \mathrm{mg} / \mathrm{L})$ demonstrating significant teratogenicity and lethality alone. In contrast to dose-dependence, there was little effect of ENF on the time-dependence of CAR toxicity (mainly within 0-48 hpf). The inclusion of ENF also altered the specific deformities induced by CAR, as body axis curvature was rare under MIX treatment (Figure 3D1). 
A

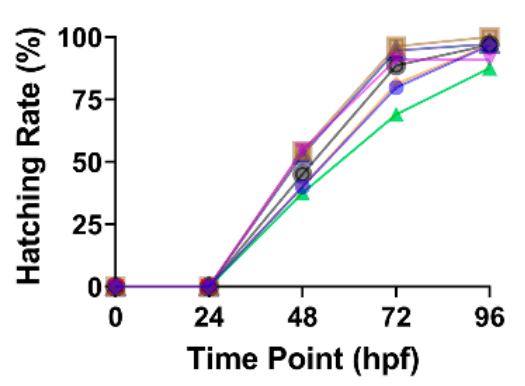

C

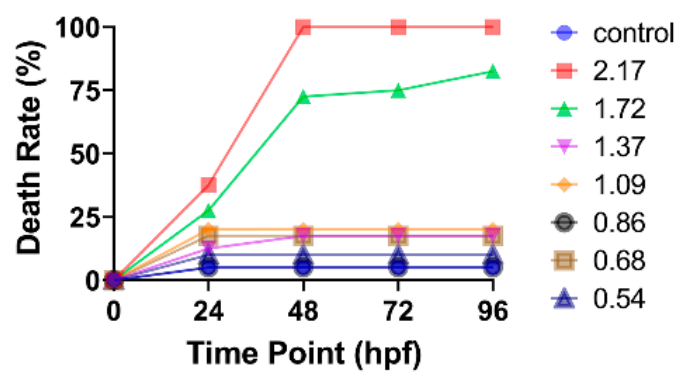

B
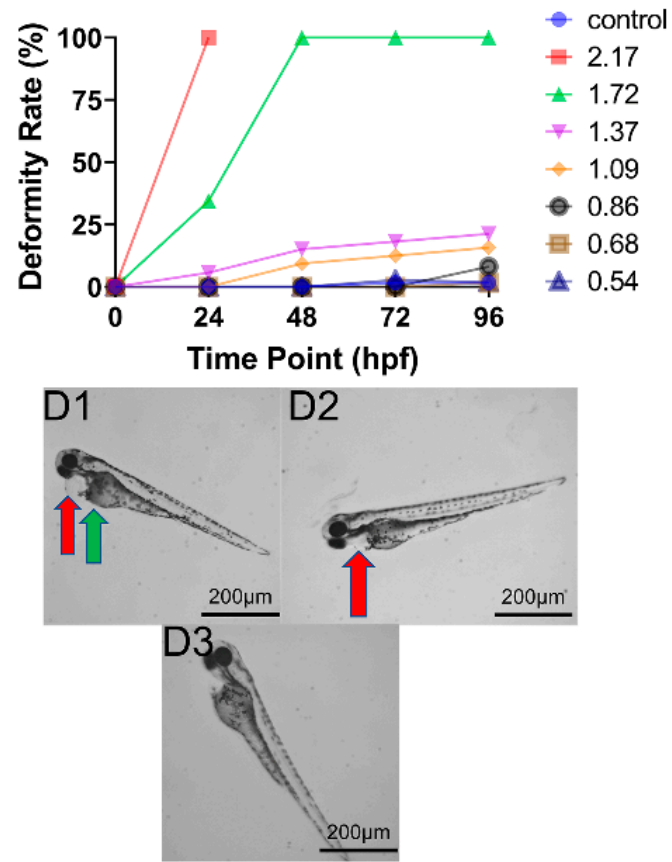

Figure 3. Effects of CAR plus ENF (MIX) on zebrafish development $(n=60)$. (A) Time-response curves of hatching rate at all tested doses. (B) Time-response curves of deformity rate at all tested doses. (C) Time-response curves of death rate at all tested doses. (D) Typical deformities induced by (D1) $1.72 \mathrm{mg} / \mathrm{L}$, (D2) $0.86 \mathrm{mg} / \mathrm{L}$, and (D3) $0.54 \mathrm{mg} / \mathrm{L} \mathrm{MIX} \mathrm{at} 96 \mathrm{hpf}$. Red arrows demarcate areas of pericardial oedema, green arrows demarcate regions of yolk sac oedema, and blue arrows indicate regions of spinal curvature.

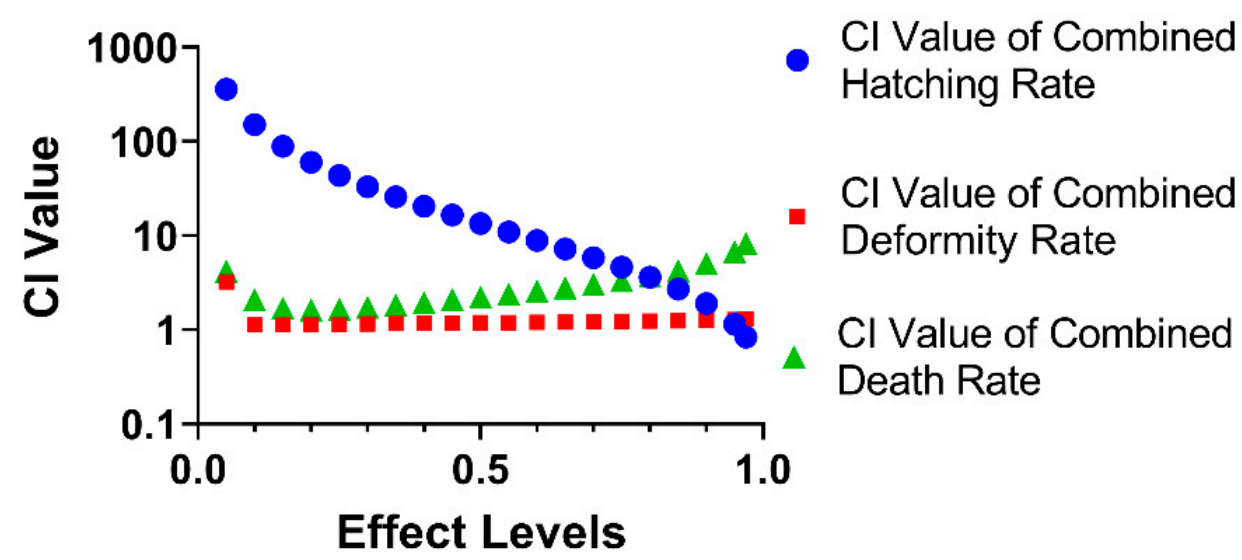

Figure 4. Combination indices (CI values) of MIX for $48 \mathrm{hpf}$ hatching rate (blue), $96 \mathrm{hpf}$ deformity rate (red), and $96 \mathrm{hpf}$ death rate (green). The CI values indicate that ENF antagonized the toxic effects of CAR on hatching rate, especially at low effect levels, and on death rate at higher effect levels.

\subsection{The Combined Effect of CAR and ENF on Developmental Parameters}

These qualitative changes in $48 \mathrm{hpf}$ hatching rate, $96 \mathrm{hpf}$ deformity rate, and $96 \mathrm{hpf}$ death rate between CAR alone and CAR in the presence of ENF (MIX) were then examined quantitatively by calculating combination indices (CI values), where a $\mathrm{CI}$ value $>1.1$ indicates antagonism and a CI value less than 0.9 indicates synergism. As shown in Figure 4, ENF antagonized the effects of CAR on the hatching rate at $48 \mathrm{hpf}$, deformity rate at $96 \mathrm{hpf}$, and death rate at $96 \mathrm{hpf}$ over most of the effect range, with synergism detected only in the $97-100 \%$ effect range for the $48 \mathrm{hpf}$ hatching rate. 


\subsection{Transcriptomics and qPCR Analysis of Toxicity Pathways}

Since ENF mainly exhibited antagonistic effects on CAR-induced developmental toxicity, transcriptomics analysis focused on differentially expressed genes (DEGs) between MIX and single compound treatment groups. In eggs treated with MIX, 37 genes were significantly upregulated and 303 downregulated compared to the CAR treatment group (Figure 5A,B2). Compared to ENF-treated eggs, MIX treatment upregulated 197 genes and downregulated 292 genes (Figure 5A,B1). The Kyoto Encyclopedia of Genes and Genomes (KEGG) pathway analysis revealed that this DEG set was enriched in genes involved in 'protein digestion and absorption', 'mineral absorption', 'carbon metabolism', 'fat metabolism', and 'other pathways' (Figure 5C), while GO enrichment identified multiple genes implicated in the 'cellular process', 'metabolic process', and 'biological regulation' (Figure 5D). Construction of a gene network based on GO enrichment revealed that multiple DEGs were involved in 'lipid catabolism' and 'small molecule metabolism' (Figure 5E). Thus, many of these DEGs regulate the metabolism of common nutrients.

The heat map and qPCR results showed that CAR and ENF have distinct effects on the expression levels of many metabolism-related genes (Figures 5F and 6). Specifically, both CAR and ENF promoted the expression of the metabolism-related gene apoa1a, while no significant changes could be found in MIX. Besides, CAR and ENF showed different impacts on the apoa1a gene. In addition, CAR and ENF showed antagonistic effects on expression levels of cytochrome (cyp) family genes cyp $2 p 9$ and cyp7a1. Both compounds slightly increased the expression of cyp7a1 when applied alone, but not when applied together. In contrast, MIX reduced the expression of cyp2p9, while neither compound alone influenced expression.

A
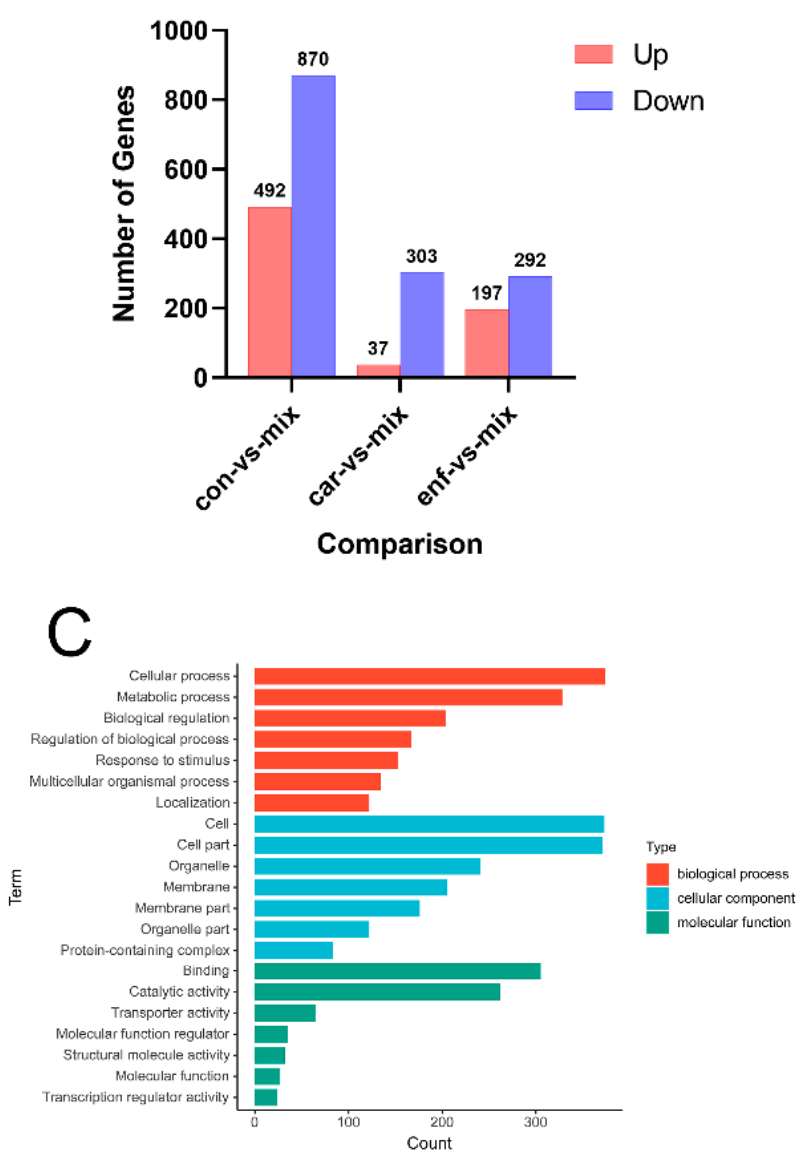

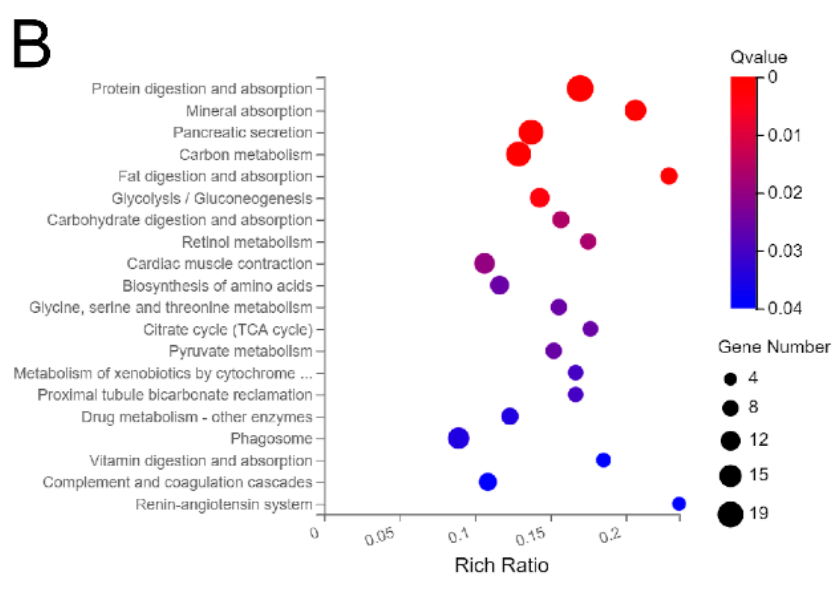

D

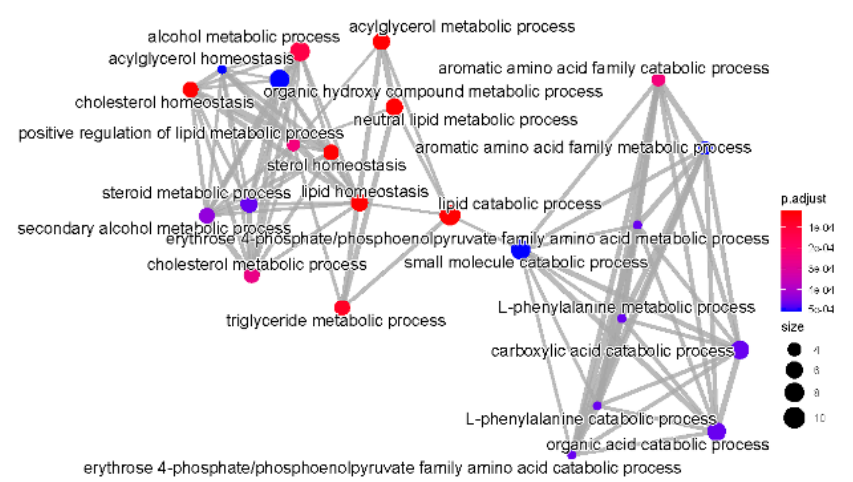

Figure 5. Cont. 


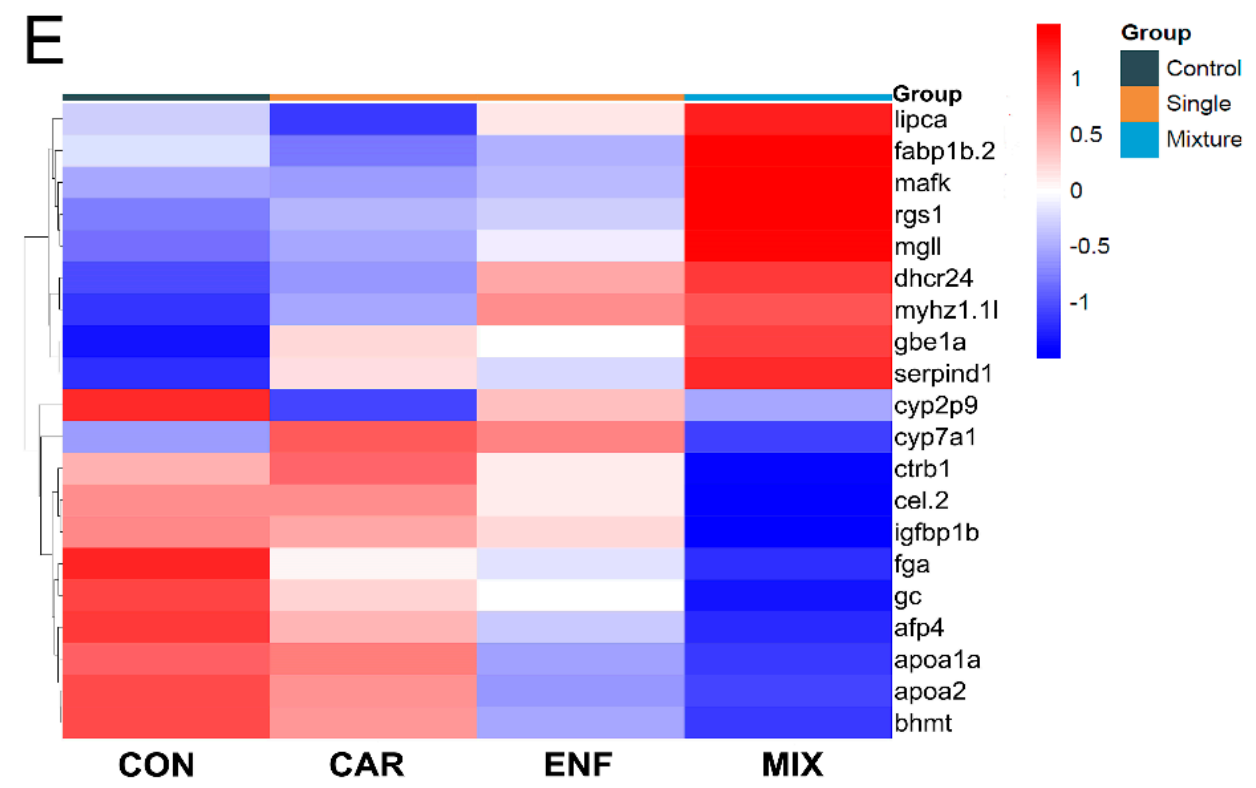

Figure 5. Transcriptomic results indicate that DEGs among treatment groups are enriched in genes required for nutrient metabolism. (A) DEGs for controls vs. MIX, CAR vs. MIX, and ENF vs. MIX at 96 hpf. (B) KEGG pathway enrichment of DEGs. The y-axis represents the significantly enriched KEGG pathways and the $x$-axis denotes the enrichment factor of DEGs. (C) GO category pattern of DEGs in 96 hpf larvae. (D) GO network of DEGs. (E) Heat map of top DEGs.

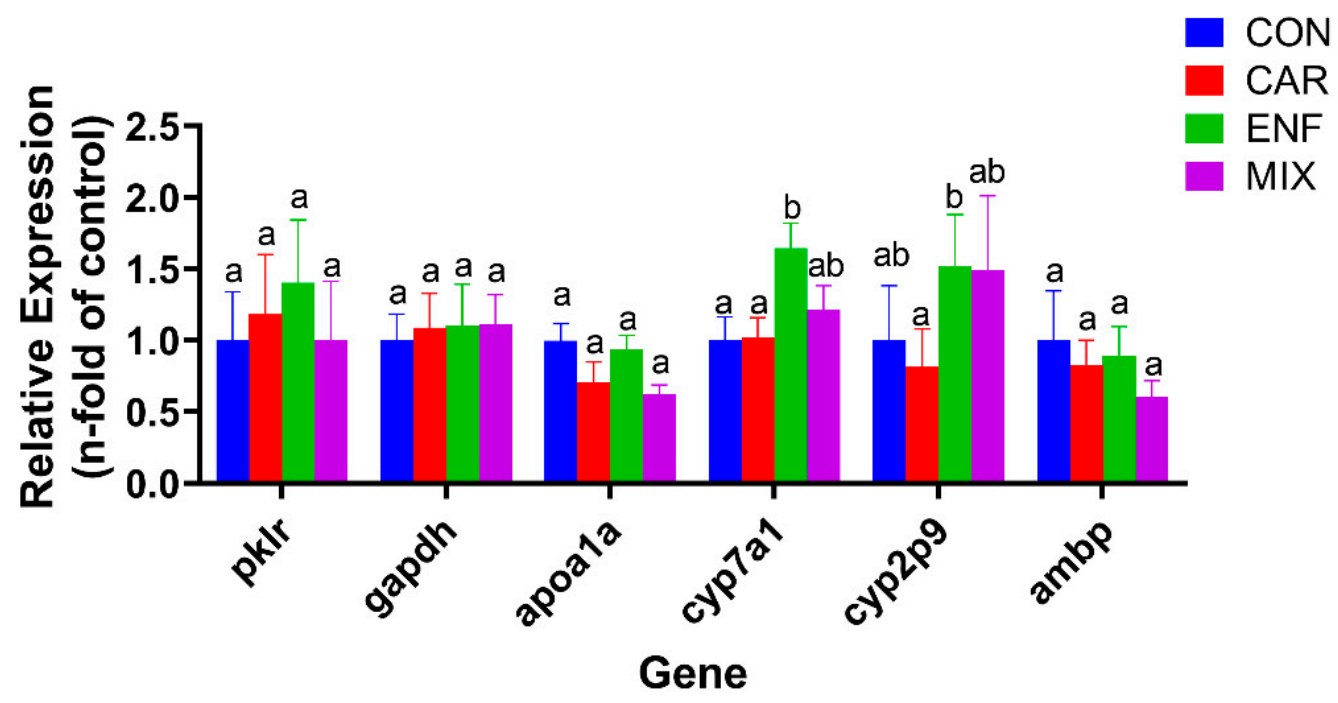

Figure 6. Relative mRNA expression levels of DEGs related to metabolism. Results are expressed as the fold-change relative to the control group. Data are shown as mean \pm SEM; (Two-way ANOVA, Tukey's HSD). Completely different lowercase letters above the bars indicate significant differences $(p<0.05)$, while any of the same lowercase letters indicate no significant difference $(p>0.05)$.

\section{Discussion}

Carbendazim residues have been detected in fruits, vegetables, and fresh grains. For example, in Loquat, Lebanon, carbendazim was detected at a maximum concentration of $0.096 \mathrm{mg} / \mathrm{kg}$ [29]. Further, the application of carbendazim to rapeseed flowers resulted in residual accumulation in the honey up to $0.35 \mathrm{mg} / \mathrm{kg}$ [30]. Carbendazim has also been detected at $0.010-0.364 \mathrm{mg} / \mathrm{kg}$ in some green tea samples from China [31]. The current study indicated that even $0.50 \mathrm{mg} / \mathrm{L}$ carbendazim can be teratogenic and lethal to zebrafish embryos. While there is no precise method to convert the residual 
amount in fresh foods to direct exposure of zebrafish, the recent increase in food safety research using zebrafish [32] and the strong correlation in toxicity parameters with rodents $(\log R=0.7340320)$ [33] does allow for a simple estimation of dose equivalence. Based on this simple calculation, $0.50 \mathrm{mg} / \mathrm{L}$ is equivalent to the rodent toxicity threshold of $9.60 \mathrm{mg} / \mathrm{L}$, which yields a theoretical ADI of $0.0096 \mathrm{mg} / \mathrm{kg}$ bw $/$ day, lower than the existing ADI (0.03 mg/kg bw/day) [34]. Thus, the actual risk of carbendazim exposure may be higher than currently believed.

Although restrictions on the administration of ENF and other antibiotics to livestock have been effective, there are still reports of excessive residue in food. For example, reported ENF residues in pork samples from selected EU countries ranged from 0.05-2.46 $\mu \mathrm{g} / \mathrm{kg}$ [35]. However, average levels detected in chicken breasts from Brazil ranged from 8.63 to $12.25 \mu \mathrm{g} / \mathrm{kg}$ [36]. These residual levels are above the ADI (allowable daily intake) evaluated by WHO (2.3 $\mu \mathrm{g} / \mathrm{kg}$ bw [37]), but below the range causing detectable toxicity in zebrafish, the substantial health risks of ENF still need attention.

Previous studies on joint CAR toxicity have focused primarily on other pesticides [38,39] and similar studies on ENF have focused on other veterinary drugs [40,41], while few studies have examined the combination of pesticides and veterinary drugs. However, as most people consume fruits, vegetables, milk, and meat every day, an examination of potential combined effects of pesticides and veterinary drugs is important for comprehensive safety assessment. Although CAR and ENF mainly exhibit antagonistic effects at the dose ranges and ratio selected, this does not mean that ENF will mitigate the health risks of CAR as mixtures can exhibit distinct combined effects (e.g., antagonism, synergy, additivity) across concentration ranges and ratios [42,43]. Such results also highlight the importance of choosing dose ranges and ratios reflective of real-world exposure for risk assessment.

Transcriptomics and qPCR identified several hundred genes differentially expressed between treatment groups, while GO and KEGG analyses indicated that many of these DEGs are involved in the absorption or utilization of nutrients. These findings are consistent with previous reports that CAR can act as a metabolic disruptor. For instance, Bao et al. found that CAR can impair glucose and lipid metabolism in the liver of adult zebrafish [44], while others have found that CAR can impair glucose, glycerol triester, and protein regulation in mice [45]. In contrast, there are no similar reports on the metabolic effects of enrofloxacin. The longer-term effects of combined exposure on metabolism warrant further study.

Genes differentially expressed between treatment groups included cyp7a1, apoa1a, and pklr. The protein product of cyp7a1, cytochrome P450 family 7 subfamily A member 1 (CYP7A1), is the rate-limiting enzyme for bile acid synthesis in the liver. Inhibition of cyp7a1 may be related to tissue-specific farnesoid $X$ receptor (Fxr) activation [46]. CYP7A1 may also be involved in the anti-hypercholesterolemia effect of plant extracts [47] and in the hepatotoxicity caused by certain anti-tuberculosis drugs [48]. Thus, abnormal expression of cyp7a1 induced by CAR or CAR plus ENF may lead to liver damage and impaired bile acid metabolism. Apoa1a (apolipoprotein A-Ia) belongs to the apolipoprotein A family and is closely related to fat metabolism and utilization. Studies have shown that the expression of apoa1 gene is affected by the acute toxicity of 3,4-dichloroaniline on zebrafish juveniles [49]. The affected expression of apoa1 was also discovered in perfluorooctanesulfonic-acidcaused abnormal lipid metabolism in adult zebrafish and offspring [50]. Like apoa1a, the product of $p k l r$, pyruvate kinase L/R (PKLR), is a glycolytic enzyme implicated in the toxicity of several other compounds. For example, the changes in HepG2 metabolism caused by bisphenol analogs were related to altered pklr expression [51]. Additionally, liver damage in Chinese rare minnows caused by carbamazepine may be related to altered pklr transcription [52]. Thus, CAR may fundamentally disrupt the glucose metabolism by altering the expression levels and enzymatic activities of APOA1A and PKLR. Again, the longer-term effects of CAR and MIX on glycolysis warrant further study. 


\section{Conclusions}

This study demonstrated that CAR and ENF reveal toxicity on the developmental parameters of zebrafish embryos when worked alone. However, ENF can antagonize the developmental toxicity of CAR in zebrafish embryos at a ratio of 1:4.82, possibly by reciprocal effects on the expression levels of metabolic enzymes, including glycolytic enzymes. The results indicate that the cross-category toxicity of different chemicals is complex and needs more research. Given the potential for combined exposure to pesticides and veterinary drugs, it is critical to document joint toxicity at realistic doses for health risk assessment.

Supplementary Materials: The following are available online at https:/ /www.mdpi.com/article/10 .3390/toxics9120349/s1, 1. mRNA Library Construction, 2. RNA-Seq Data Analyze, Supplementary Table S1. Primer pairs of selected genes in qRT-PCR analysis.

Author Contributions: Conceptualization, R.F.; methodology, R.F., W.Z.; validation, L.J., Y.L. (Ying Liu); formal analysis, R.F., W.Z.; investigation, R.F., L.J.; resources, X.Y.; data curation, S.L., Y.J.; writing—original draft preparation, R.F.; writing—review \& editing, X.Y., Y.C.; visualization, S.L., Y.L. (Yongchen Li); supervision, X.Y., Y.C.; project administration, X.Y., Y.C.; funding acquisition, X.Y., Y.C. All authors have read and agreed to the published version of the manuscript.

Funding: This research was funded by Ministry of Science and Technology of the People's Republic of China, grant number 2018YFC1603002.

Institutional Review Board Statement: Not applicable.

Informed Consent Statement: Not applicable.

Data Availability Statement: The transcriptome raw data have been submitted to NCBI database with the BioProject number PRJNA780808.

Acknowledgments: We thank openbiox community and Hiplot team (https://hiplot.com.cn, accessed on 5 October 2021) for providing technical assistance and valuable tools for data analysis and visualization.

Conflicts of Interest: The authors declare no conflict of interest.

\section{References}

1. Teglia, C.M.; Guinez, M.; Culzoni, M.J.; Cerutti, S. Determination of residual enrofloxacin in eggs due to long term administration to laying hens. Analysis of the consumer exposure assessment to egg derivatives. Food Chem. 2021, 351, 8. [CrossRef] [PubMed]

2. Ji, X.; Xu, Y.; Wang, J.; Lyu, W.; Li, R.; Tan, S.; Xiao, Y.; Tang, B.; Yang, H.; Qian, M. Multiresidue determination of antibiotics in ready-to-eat duck eggs marketed through e-commerce stores in China and subsequent assessment of dietary risks to consumers. J. Food Sci. 2021, 86, 2145-2162. [CrossRef]

3. Ellerbrock, R.E.; Canisso, I.F.; Roady, P.J.; Rothrock, L.T.; Zhong, L.; Wilkins, P.; Dirikolu, L.; Lima, F.S.; Honoroto, J. Diffusion of enrofloxacin to pregnancy fluids and effects on fetal cartilage after intravenous administration to late pregnant mares. Equine Vet. J. 2019, 51, 544-551. [CrossRef] [PubMed]

4. Liu, J.; Chen, Z.; Li, Y.; Liu, Y. Effects of low concentration enrofloxacin on SPF mice intestinal microflora. Sci. Agric. Sin. 2005, 38, 1905-1910.

5. Xu, X.; Chen, J.; Li, B.; Tang, L. Carbendazim residues in vegetables in China between 2014 and 2016 and a chronic carbendazim exposure risk assessment. Food Control 2018, 91, 20-25. [CrossRef]

6. Songür, S.H.; Koçkaya, E.A.; Selmanoĝlu, G.; Barlas, N. Dose-dependent effects of carbendazim on rat thymus. Cell Biochem. Funct. 2005, 23, 457-460. [CrossRef] [PubMed]

7. Matsuo, F.; Nakai, M.; Nasu, T. The fungicide carbendazim induces meiotic micronuclei in the spermatids of the rat testis. J. Vet. Med. Sci. 1999, 61, 573-576. [CrossRef]

8. Zhang, Y.H.; Liu, S.S.; Liu, H.L.; Liu, Z.Z. Evaluation of the combined toxicity of 15 pesticides by uniform design. Pest Manag. Sci. 2010, 66, 879-887. [CrossRef] [PubMed]

9. Hernandez, A.F.; Parron, T.; Tsatsakis, A.M.; Requena, M.; Alarcon, R.; Lopez-Guarnido, O. Toxic effects of pesticide mixtures at a molecular level: Their relevance to human health. Toxicology 2013, 307, 136-145. [CrossRef] [PubMed]

10. Gonzalez-Pleiter, M.; Gonzalo, S.; Rodea-Palomares, I.; Leganes, F.; Rosal, R.; Boltes, K.; Marco, E.; Fernandez-Pinas, F. Toxicity of five antibiotics and their mixtures towards photosynthetic aquatic organisms: Implications for environmental risk assessment. Water Res. 2013, 47, 2050-2064. [CrossRef] [PubMed] 
11. Eguchi, K.; Nagase, H.; Ozawa, M.; Endoh, Y.S.; Goto, K.; Hirata, K.; Miyamoto, K.; Yoshimura, H. Evaluation of antimicrobial agents for veterinary use in the ecotoxicity test using microalgae. Chemosphere 2004, 57, 1733-1738. [CrossRef] [PubMed]

12. Zhang, Y.; Liu, H.; Wang, S.; Ding, T.; Xu, N.; Yu, K.; Shen, W.; Zhao, Z.; Liu, Y.; Wu, B.; et al. Simultaneous Determination of Eight Kinds of Drug Residues in Sprouts by High Performance Liquid Chromatography—Tandem Mass Spectrometry. J. Instrum. Anal. 2015, 34, 164-170.

13. Gong, Y.; Zou, X.; Zhang, L.; Wu, Y.; Dai, M.; Chen, Z.; Zhang, M. Study on detecting enrofloxacin residues in food by chemiluminescence immunoassay. Sci. Technol. Food Ind. 2014, 35, 66.

14. Jagadeeswaran, P. Zebrafish: A tool to study hemostasis and thrombosis. Curr. Opin. Hematol. 2005, 12, 149-152. [CrossRef] [PubMed]

15. Hill, A.J.; Teraoka, H.; Heideman, W.; Peterson, R.E. Zebrafish as a model vertebrate for investigating chemical toxicity. Toxicol. Sci. 2005, 86, 6-19. [CrossRef]

16. Chen, H.; Chen, K.; Qiu, X.C.; Xu, H.; Mao, G.H.; Zhao, T.; Feng, W.W.; Okeke, E.S.; Wu, X.Y.; Yang, L.Q. The reproductive toxicity and potential mechanisms of combined exposure to dibutyl phthalate and diisobutyl phthalate in male zebrafish (Danio rerio) Chemosphere 2020, 258, 13. [CrossRef] [PubMed]

17. Li, Y.N.; Zhang, Q.N.; Fang, J.; Ma, N.; Geng, X.; Xu, M.; Yang, H.; Jia, X.D. Hepatotoxicity study of combined exposure of DEHP and ethanol: A comprehensive analysis of transcriptomics and metabolomics. Food Chem. Toxicol. 2020, 141, 9. [CrossRef] [PubMed]

18. Gan, J.; Wu, Y.; Li, S.; Su, B. Effects of different concentrations of dimethylsulfoxide on development of zebrafish embryos and dopamine neurons. J. Third Mil. Med. Univ. 2019, 41, 1538-1544.

19. Chen, T.H.; Wang, Y.H.; Wu, Y.H. Developmental exposures to ethanol or dimethylsulfoxide at low concentrations alter locomotor activity in larval zebrafish: Implications for behavioral toxicity bioassays. Aquat. Toxicol. 2011, 102, 162-166. [CrossRef]

20. Ministry of Agriculture and Rural Affairs of the People's Republic of China. GB 2763-2021 National Food Safety Standard-Maximum Residue Limits of Pesticides in Food; MOA: Beijing, China, 2021.

21. Ministry of Agriculture and Rural Affairs of the People's Republic of China. GB 31650-2019 National Food Safety StandardMaximum Residue Limits for Veterinary Drugs in Foods; MOA: Beijing, China, 2019.

22. Cock, P.J.A.; Fields, C.J.; Goto, N.; Heuer, M.L.; Rice, P.M. The Sanger FASTQ file format for sequences with quality scores, and the Solexa/Illumina FASTQ variants. Nucleic Acids Res. 2010, 38, 1767-1771. [CrossRef]

23. Kim, D.; Landmead, B.; Salzberg, S.L. HISAT: A fast spliced aligner with low memory requirements. Nat. Methods 2015, 12, 357-360. [CrossRef] [PubMed]

24. Langmead, B.; Salzberg, S.L. Fast gapped-read alignment with Bowtie 2. Nat. Methods 2012, 9, 357-359. [CrossRef]

25. Li, B.; Dewey, C.N. RSEM: Accurate transcript quantification from RNA-Seq data with or without a reference genome. BMC Bioinform. 2011, 12, 323. [CrossRef]

26. Love, M.I.; Huber, W.; Anders, S. Moderated estimation of fold change and dispersion for RNA-seq data with DESeq2. Genome Biol. 2014, 15, 550. [CrossRef]

27. Davis, J.A.; Gift, J.S.; Zhao, Q.J. Introduction to benchmark dose methods and US EPA's benchmark dose software (BMDS) version 2.1.1. Toxicol. Appl. Pharmacol. 2011, 254, 181-191. [CrossRef] [PubMed]

28. Chou, T.C. Preclinical versus clinical drug combination studies. Leuk. Lymphoma 2008, 49, 2059-2080. [CrossRef]

29. Zeid, M.I.A.; Awad, M.K.; Melki, K.C.; Jawdah, Y.A.; Jammoul, A.M. Pesticides residues on Loquat: A minor crop in Lebanon. Food Control 2021, 130, 108297. [CrossRef]

30. Li, Y.H.; Zhou, B.L.; Qian, M.R.; Wang, Q.; Zhang, H. Transfer Assessment of Carbendazim Residues from Rape Flowers to Apicultural Products. J. Anal. Methods Chem. 2017, 2017, 7. [CrossRef]

31. Zhou, L.; Jiang, Y.P.; Lin, Q.; Wang, X.; Zhang, X.Z.; Xu, J.; Chen, Z.M. Residue transfer and risk assessment of carbendazim in tea. J. Sci. Food Agric. 2018, 98, 5329-5334. [CrossRef] [PubMed]

32. Bailone, R.L.; Aguiar, L.K.d.; Roca, R.d.O.; Borra, R.C.; Corrêa, T.; Janke, H.; Fukushima, H.C.S. Zebrafish as an animal model for food safety research: Trends in the animal research. Food Biotechnol. 2019, 33, 283-302. [CrossRef]

33. Ali, S.; van Mil, H.G.J.; Richardson, M.K. Large-Scale Assessment of the Zebrafish Embryo as a Possible Predictive Model in Toxicity Testing. PLoS ONE 2011, 6, e21076. [CrossRef] [PubMed]

34. World Health Organization. Evaluation of Data for Acceptable Daily Intake (ADI) for Humans, Maximum Residue Levels and Supervised Trials Median Residues (STMRs); WHO: Geneva, Switzerland, 2004.

35. Bartkiene, E.; Ruzauskas, M.; Bartkevics, V.; Pugajeva, I.; Zavistanaviciute, P.; Starkute, V.; Zokaityte, E.; Lele, V.; Dauksiene, A.; Grashorn, M.; et al. Study of the antibiotic residues in poultry meat in some of the EU countries and selection of the best compositions of lactic acid bacteria and essential oils against Salmonella enterica. Poult. Sci. 2020, 99, 4065-4076. [CrossRef]

36. Panzenhagen, P.H.; Aguiar, W.S.; Gouvêa, R.; de Oliveira, A.M.; Barreto, F.; Pereira, V.L.; Aquino, M.H. Investigation of enrofloxacin residues in broiler tissues using ELISA and LC-MS/MS. Food Addit. Contam. Part A Chem. Anal. Control Expo. Risk Assess. 2016, 33, 639-643. [CrossRef]

37. World Health Organization. Evaluation of Certain Veterinary Drug Residues in Food; WHO: Geneva, Switzerland, 1998.

38. Lu, S.-Y.; Liao, J.-W.; Kuo, M.-L.; Hwang, J.-S.; Ueng, T.-H. Antagonistic and synergistic effects of carbendazim and flutamide exposures in utero on reproductive and developmental toxicity in rats. J. Food Drug Anal. 2006, 14, 120-132. [CrossRef] 
39. Dikic, D.; Mojsovic-Cuic, A.; Cupor, I.; Benkovic, V.; Horvat-Knezevic, A.; Lisicic, D.; Orsolic, N. Carbendazim combined with imazalil or cypermethrin potentiate DNA damage in hepatocytes of mice. Hum. Exp. Toxicol. 2012, 31, 492-505. [CrossRef] [PubMed]

40. Wang, G.X.; Zhang, Q.; Li, J.L.; Chen, X.Y.; Lang, Q.L.; Kuang, S.P. Combined effects of erythromycin and enrofloxacin on antioxidant enzymes and photosynthesis-related gene transcription in Chlorella vulgaris. Aquat. Toxicol. 2019, 212, 138-145. [CrossRef] [PubMed]

41. Luan, Y.H.; Zhao, J.J.; Han, H.F.; Shen, J.Z.; Tang, S.S.; Cheng, L.L. Toxicologic effect and transcriptome analysis for short-term orally dosed enrofloxacin combined with two veterinary antimicrobials on rat liver. Ecotoxicol. Environ. Saf. 2021, 220, 10. [CrossRef] [PubMed]

42. Zhang, C.; Chen, Y. Acute toxicity and joint toxicity of $\mathrm{Cr} \sim(6+), \mathrm{Mn} \sim(7+)$ and $\mathrm{Zn} \sim(2+)$ on Palaemon carincauda juvenile. Mar. Environ. Sci. 2013, 32, 235-238.

43. Wei, F.; Wang, D.; Li, H.; You, J. Joint toxicity of imidacloprid and azoxystrobin to Chironomus dilutus at organism, cell, and gene levels. Aquat. Toxicol. 2021, 233, 105783. [CrossRef]

44. Bao, Z.; Zhao, Y.; Wu, A.; Lou, Z.; Lu, H.; Yu, Q.; Fu, Z.; Jin, Y. Sub-chronic carbendazim exposure induces hepatic glycolipid metabolism disorder accompanied by gut microbiota dysbiosis in adult zebrafish (Daino rerio). Sci. Total Environ. 2020, 739, 140081. [CrossRef] [PubMed]

45. Farag, A.; Ebrahim, H.; ElMazoudy, R.; Kadous, E. Developmental Toxicity of Fungicide Carbendazim in Female Mice. Birth Defects Res. Part B Dev. Reprod. Toxicol. 2011, 92, 122-130. [CrossRef]

46. Kong, B.; Wang, L.; Chiang, J.Y.L.; Zhang, Y.C.; Klaassen, C.D.; Guo, G.L. Mechanism of tissue-specific farnesoid X receptor in suppressing the expression of genes in bile-acid synthesis in mice. Hepatology 2012, 56, 1034-1043. [CrossRef]

47. Wang, Y.; Han, Y.L.; Chai, F.N.; Xiang, H.M.; Huang, T.; Kou, S.M.; Han, B.; Gong, X.B.; Ye, X.L. The antihypercholesterolemic effect of columbamine from Rhizoma Coptidis in HFHC-diet induced hamsters through HNF-4 alpha/FTF-mediated CYP7A1 activation. Fitoterapia 2016, 115, 111-121. [CrossRef]

48. Sharma, R.; Kaur, R.; Mukesh, M.; Sharma, V.L. Assessment of hepatotoxicity of first-line anti-tuberculosis drugs on Wistar rats. Naunyn-Schmiedebergs Arch. Pharmacol. 2018, 391, 83-93. [CrossRef] [PubMed]

49. Vieira, L.R.; Hissa, D.C.; de Souza, T.M.; Sa, C.A.; Evaristo, J.A.M.; Nogueira, F.C.S.; Carvalho, A.F.U.; Farias, D.F. Proteomics analysis of zebrafish larvae exposed to 3,4-dichloroaniline using the fish embryo acute toxicity test. Environ. Toxicol. 2020, 35, 849-860. [CrossRef]

50. Cui, Y.; Lv, S.; Liu, J.; Nie, S.; Chen, J.; Dong, Q.; Huang, C.; Yang, D. Chronic perfluorooctanesulfonic acid exposure disrupts lipid metabolism in zebrafish. Hum. Exp. Toxicol. 2017, 36, 207-217. [CrossRef] [PubMed]

51. Yue, S.; Yu, J.; Kong, Y.; Chen, H.; Mao, M.; Ji, C.; Shao, S.; Zhu, J.; Gu, J.; Zhao, M. Metabolomic modulations of HepG2 cells exposed to bisphenol analogues. Environ. Int. 2019, 129, 59-67. [CrossRef] [PubMed]

52. Yan, S.; Wang, M.; Liang, X.; Martyniuk, C.J.; Zha, J.; Wang, Z. Environmentally relevant concentrations of carbamazepine induce liver histopathological changes and a gender-specific response in hepatic proteome of Chinese rare minnows (Gobiocypris rarus). Environ. Pollut. 2018, 243, 480-491. [CrossRef] 